\title{
Modèle intégré pluie - eau de surface - eau souterraine
}

\author{
An integrated rainfall, \\ surface and underground runoff model
}

\author{
Georges Girard *, Emmanuel Ledoux * * \\ et Jean-Pierre Villeneuve ** \\ * Office de la Recherche Scientifique et Technique d'Outre-Mer (ORSTOM) \\ ** École Nationale Supérieure des Mines de Paris \\ ** Institut National de la Recherche Scientifique du Québec (INRS-Eau)
}

Le modèle qui va être présenté résulte d'une association du modèle hydrologique à discrétisation spatiale mis au point lors d'une coopération INRS-Eau-ORSTOM et du modèle de simulation des écoulements souterrains en système multicouche, NEWSAM, conçu à l'Ecole des Mines de Paris.

L'objectif d'un tel modèle est la simulation numérique des écoulements sur un système hydrologique comprenant un nombre quelconque de bassins versants et d'aquifères, en tentant une représentation intégrée des des différents aspects du cycle de l'eau depuis les précipitations jusqu'aux débits aux exutoires.

Lc présent article fait état des recherches en cours menées en collaboration par l'INRS-Eau, l'ORSTOM et l'Ecole des Mines de Paris, en fournissant une description des différents principes physiques contenus dans le modele ainsi qu'une tentative d'application sur le bassin crayeux de la Lys dans le Nord de la France.

\section{Principe du modèle}

Le cycle de l'eau est constitué de nombreux mécanismes complexes ayant des intéractions les uns sur les autres. La caractéristique fondamentale du modèle est tout d'abord de décomposer la schématisation du système hydrologique en étapes indépendantes et dont les résultats soient contrôlables séparément. On réunit donc à la fois les avantages évidents d'une simulation globale d'un système hydrologique, ainsi que la possibilité de vérification de la validité de certains mécanismes internes souvent traités séparément car ils relèvent de spécialités distinctes dans les sciences de l'eau.
Le principe de la modélisation consiste en une généralisation de la schématisation multicouche classique en hydrogéologie. Le modèle distingue d'une part une couche supérieure, dite de surface où s'effectue le par. tage de l'eau disponible, en eau de surface et en infiltration, ainsi que le transfert superficiel, et d'autre part, un nombre variable de couches profondes, sièges d'écoulements souterrains, éventuellement interconnectées entre elles, figurant la succession verticale des niveaux aquifères telle qu'on peut la rencontrer en hydrogéologie.

Chacune de ces couches est ensuite discrétisée en mailles auxquelles sont attribués les différents parametres du système et sur lesquelles s'effectueront les transferts d'eau.

L'ensemble est articulé, dans l'état actuel, en quatre programmes écrits en langage FORTRAN, destinés à općrer séquentiellement.

- Le programme GEOCOU organise en fonction des données physiographiques la discrétisation spatiale du milieu souterrain, ainsi que l'arbre de drainage des bassins en surface.

- Le programme MODSUR évalue pour chaque maille de surface la répartition entre l'eau disponible pour le ruissellement et pour l'infiltration à partir des données météorologiques en fonction des paramètres attribués au sol.

- Le programme NONSAT assure le transfert de l'infiltration nette calculée dans MODSUR en alimentation de la nappe, suivant un processus uniforme par groupement de mailles constituant des zones homogènes pour les caractéristiques du milieu non saturé.

- Le programme MODCOU simule finalement les transferts de l'eau à la fois dans la couche de surface et dans les couches souterraines, en tenant compte 
d'interactions à double sens entre les nappes et les rivières.

Nous allons maintenant donner pour chacun de ces programmes une description sommaire des mécanismes qui y sont représentés ainsi que des moyens de contrôle de la simulation.

\section{Programme GEOCOU}

Ce programme est organisé autour des données suivantes :

\section{Définition du maillage}

La discrétisation initiale est constituée par un réseau de mailles carrées de côté $8 a$. Localement ce maillage peut être subdivisé en sous maillages de côté $4 a, 2 a$ ou a. La discrétisation de certains secteurs des nappes et de la couche de surface peut ainsi être raffinée puisqu'on dispose de quatre tailles de mailles. Cette subdivision doit s'effectuer graduellement dans l'espace de telle sorte qu'une maille donnée ne puisse avoir pour voisine qu'une maille de superficie soit identique, soit quatre fois plus grande, soit encore quatre fois plus petite, conformé. ment à l'exemple suivant :
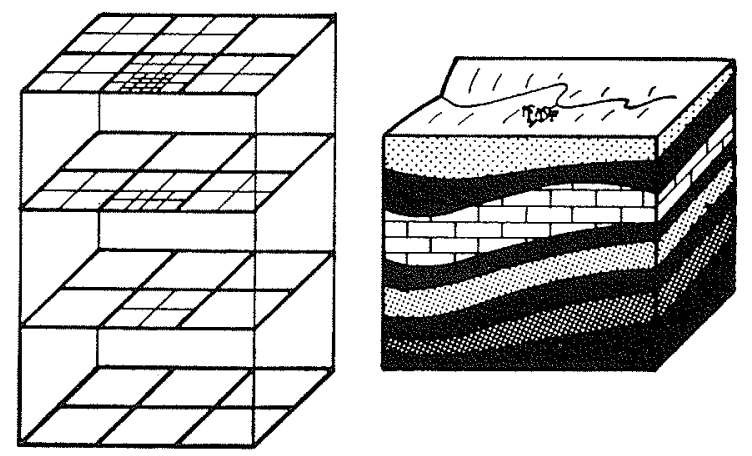

Chaque maille ainsi définie est ensuite numérotée en séquence, indépendamment de sa taille et en commençant à un pour chacune des couches. Le repérage des mailles voisines de chaque maille est assuré automatiquement dans chaque couche et d'une couche à l'autre par le programme à partir de données décrivant la position de chaque maille par rapport à la discrétisation initiale.

\section{Définition du réseau de drainage de surface}

L'arborescence du réseau de drainage de surface est définie par la connaissance pour chaque maille d'une direction unique de vidange choisie suivant les huit directions possibles du plan, compte tenu du type de maillage utilisé et des données topographiques. Les mailles dont la vidange s'effectue à l'extérieur du domaine constituent des exutoires potentiels.

Ayant choisi parmi ces exutoires ceux dont on désire une simulation des débits on sélectionne en remontant de l'aval vers l'amont les mailles figurant le réseau hydrographique principal ou "mailles rivières". (cf. schéma).

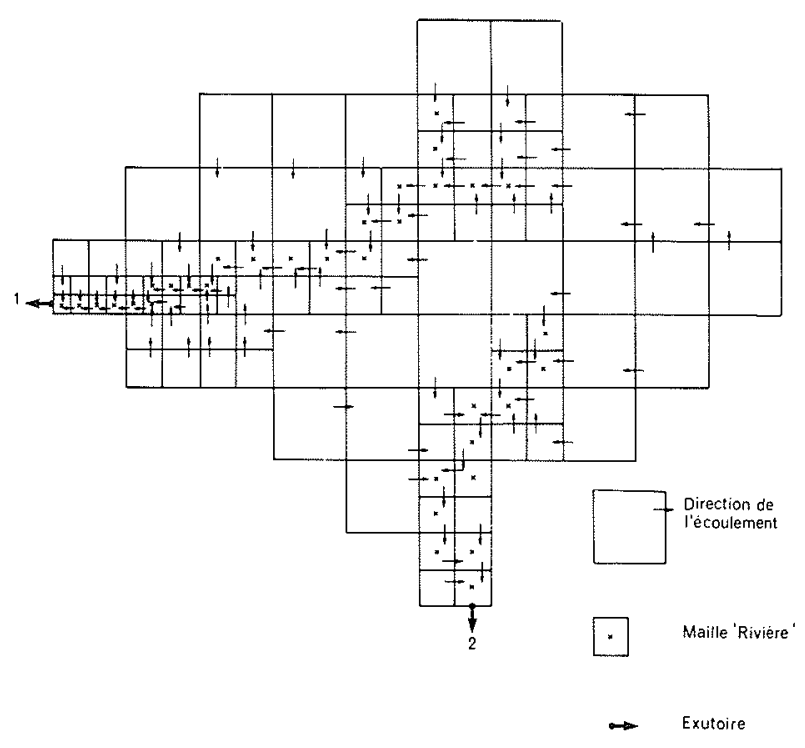

A chacun des exutoires ainsi retenus se trouve affecté un bassin, sur lequel aura lieu le transfert de surface. Pour chaque maille rivière figurant dans les bassins on recherche dans le réseau de drainage la liste des mailles participant à son alimentation à l'exception des autres mailles rivières. On définit ainsi autant de sous-bassins qu'il y a de mailles rivières dont les écoulements seront traités individuellement.

\section{Définition des données physiographiques}

Ces données concernent ici uniquement les mailles de la couche de surface. Elles comprennent dans l'état actuel pour chaque maille :

- des données altimétriques qui seront utilisées d'une part pour le calcul du temps de transfert de l'eau de surface sur les mailles, d'autre part pour évaluer l'épaisseur de la zone non saturée, enfin, éventuellement pour une modulation des précipitations ;

- des données exprimées en pourcentage de la superficie de chaque maille définissant des zones homogènes sur lesquelles seront calculés les bilans hydriques dans le programme MODSUR. Ces zones seront choisies en fonction de critères adaptés aux problèmes qui pourront provenir par exemple des caractéristiques des sols ou de la couverture végétale.

Les surfaces de plan d'eau libre serviront au calcul des coefficients de vidange entre "mailles rivières" intervenant dans les transferts.

L'ensemble de ces données est soumis à des contrôles de compatibilité avant traitement, puis récapitulé sous forme de tableaux et de plans. Les résultats sont finalement stockés sur fichier pour être transmis aux programmes suivants.

\section{Programme MODSUR}

L'objectif du programme est de fournir en fonction du temps sur la couche de surface la répartition de l'eau disponible pour le ruissellement dans le réseau hydrogra- 
phique principal, et pour l'infiltration vers les couches profondes.

Ce programme nécessite les données suivantes :

\section{Données météorologiques}

- Les précipitations (pluie et eau de fonte de neige) doivent être fournies avec un pas de temps régulier sur un ensemble de zones pluviométriques homogènes recouvrant la couche de surface. Ces zones homogènes sont choisies d'après les caractéristiques pluviométriques régionales et introduites dans le modèle en attribuant un numéro de zone à chacune des mailles de la surface.

- Les évapotranspirations potentielles sont dans l'état actuel supposées homogènes sur l'ensemble du domaine et introduites avec un pas de temps également régulier (mois, décade. . .).

\section{Données caractérisant les fonctions productions}

Le rôle des fonctions productions est de répartir la lame d'eau précipitée entre ruissellement, infiltration et évapotranspiration. Ce calcul est mené à chaque pas de temps des données météorologiques, sur chacune des zones pluviométriques homogènes et pour différentes caractéristiques de fonctions productions, indépendamment du maillage de la couche surface. Chaque fonction production fonctionne suivant un mécanisme adapté au problème établi par l'opérateur et reste spécifique de la zone homogène déterminée au moment de la discrétisation spatiale de la surface. Chaque fonction production possède un nombre limité à 10 de paramètres fixés par 1'opérateur.

\section{Données caractérisant les temps de concentration des bassins versants}

Un temps de concentration est attribué à chaque bassin versant. Ce temps désigne la durée du plus long parcours sur le bassin et permet ainsi de classer les mailles figurant dans l'arbre d'écoulement en zones isochrones par rapport à l'exutoire de chaque sous-bassin. Plusieurs essais peuvent être réalisés avec différents temps, pour un même calcul.

Ce programme MODSUR assure chronologiquement les opérations suivantes:

outre la répartition de la lame d'eau précipitée vue précédemment.

a) l'évaluation globale des termes du bilan d'eau sur chacune des zones pluviométriques et des zones de production. L'examen des valeurs de chacun de ces termes permet d'apprécier l'adéquation de chacune des fonctions productions introduites et la validité des paramètrès utilisés ;

b) l'évaluation globale des termes du bilan d'eau sur chaque sous-bassin versant et bassin étudié permet d'effectuer des comparaisons avec les termes du bilan propre du bassin ;

c) la détermination, pour chaque maille de la couche de surface et chaque pas de temps calcul du volume de ruissellement selon le pourcentage de chaque zone de production sur cette maille et son attachement à une zone pluviométrique homogène, ainsi que le volume d'infiltration nette vers le souterrain. Au volume de ruissellement est ajouté le volume infiltré pour les mailles de surface n'ayant pas de correspondantes dans les couches du souterrain ;

d) la sommation, après transfert par isochronisme des volumes ruisselés sur toutes les mailles d'un sous-bassin versant, attaché à la maille "rivière" exutoire de ce sousbassin, et ce, pour tous les sous-bassins;

e) la mémorisation à chaque pas de temps calcul des débits ruisselés sur les sous-bassins et des volumes infiltrés sur les mailles de surface, qui seront utilisés ultérieurement dans le programme MODCOU ;

f) l'évaluation du débit dû au seul ruissellement aux exutoires des bassins par un second transfert isochrone sur les mailles "rivières" de ces bassins.

Au terme du programme MODSUR deux types de contrôle sont ainsi possibles:

- appréciation du bilan hydrologique global pour chaque bassin et pour diverses périodes de simulation permettant d'intervenir sur les paramètres des fonctions productions;

- évaluation des temps de concentration des bassins par recherche du synchronisme entre la composante ruissellement des crues calculées et les crues observées.

\section{Programme NONSAT}

Ce programme assure en l'état actuel, le transfert d'eau de l'infiltration nette calculée précédemment au niveau de la nappe au moyen d'une série de $N$ réservoirs linéaires ayant le même temps de délai comparable au modèle de NASH. Le nombre de réservoirs et le temps de délai, pour chaque zone homogène du sol non saturé, sont choisis par l'opérateur selon ses propres connaissances.

Les graphiques de variations de l'alimentation de la nappe dans le temps pour des mailles pré-choisies permettent de visualiser l'influence de ces transferts.

\section{Programme MODCOU}

Ce programme constitue le modèle couplé eau de surface-eau souterraine proprement dit.

Il effectue le calcul des transferts de l'eau de surface et de l'eau souterraine, ainsi que celui de leur interaction, suivant un pas de temps choisi par l'utilisateur comme multiple ou sous multiple du pas de temps des données météorologiques.

La période totale de simulation est organisée en phase de calcul servant à l'introduction de données lentement variables et à des contrôles intermédiaires.

Les données requises sont les suivantes:

1) Données hydrologiques (discrétisées sur les mailles rivières)

- volumes d'eau initiaux dans le réseau hydrogragraphique,

- paramètres définissant les coefficients de tarissement. 
2) Données hydrogéologiques (discrétisées sur les mailles des couches souterraines)

- piézométrie initiale,

- transmissivités, coefficients de drainage, coefficients d'emmagasinement, etc.,

- prélèvements et apport dans les nappes autres que l'infiltration,

- conditions aux limites des nappes.

3) Données caractérisant les relations nappe-rivière (discrétisées sur les mailles souterraines situées sur le tracé des rivières)

- niveau de drainage des nappes dans la couche de surface,

- coefficients de transfert nappe-rivière,

- infiltration maximale en rivière.

4) Données concernant le calcul numérique

- durée de la simulation

- durée du pas de temps de calcul

- critères de convergence, etc.

5) Données concernant l'édition des résultats

- édition sous forme de tableau des paramètres hy. drologiques introduits sur chaque maille (en option)
- bilan hydraulique souterrain au dernier pas de temps - cartes piézométriques, etc.

La simulation consiste en trois opérations répétées à chaque pas de temps calcul.

- Calcul du transfert souterrain, exécuté par intégration numérique des équations de l'écoulement en milieu poreux discrétisées sur les mailles des couches profondes.

- Evaluation des débits échangés entre les nappes et le réseau hydrographique. Ces débits sont calculés sur les mailles en relation avec la rivière conformément au schéma suivant :

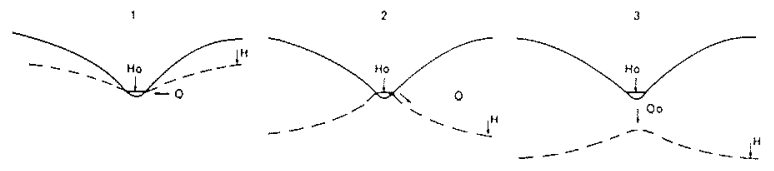

- Situations 1 et 2 : rivière et nappe connectées avec

$Q=T P\left(H-H_{0}\right)$

$Q \quad$ débit fourni à la rivière

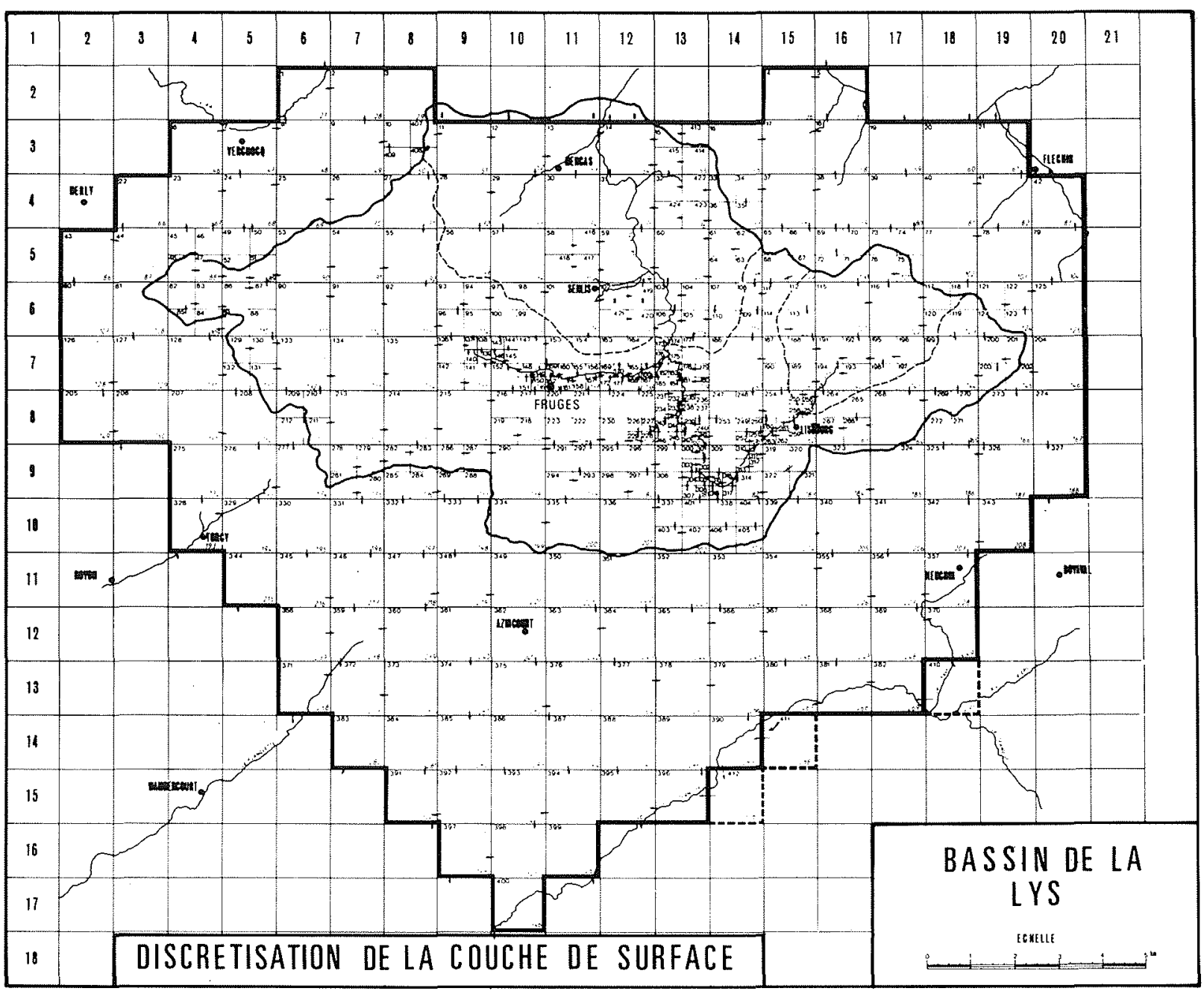

Figure 1 - Discrétisation de la couche de surface. Schéma du réseau d'écoulement. 
$T P$ coefficient de transfert nappe-rivière

$H$ niveau piézométrique de la nappe

$H_{0} \quad$ niveau de la rivière.

- Situation 3 : rivière et nappe déconnectées

$Q=Q_{0}$

$Q_{0}$ représente le débit maximal infiltrable limité soit par les possibilités de transfert à travers la zone non saturée, soit par le débit disponible en rivière.

- Calcul du transfert de surface. Ce calcul est mené de l'aval vers l'amont par vidanges de zones isochrones obtenues par regroupement en biefs des mailles rivières contiguës situées à un nombre entier de pas de temps calcul de l'exutoire ou d'une station hydrométrique.

Le coefficient de tarissement d'un bief à l'autre est choisi égal au plus grand coefficient de tarissement des mailles formant le bief.

En fin de programme sont édités:

- les courbes de variation des débits aux exutoires des bassins versants et aux stations hydrométriques,

- les courbes de variation des niveaux piézométriques sur certaines mailles choisies.

\section{Conclusion. Tentative d'application}

Le modèle qui vient d'être décrit est actuellement soumis à des tests de contrôle. Un essai est en cours sur l'exemple du bassin de la Lys dans le Nord de la France.

A l'occasion d'une récente étude réalisée pour le compte de l'Agence de l'Eau Artois-Picardie on disposait de données météorologiques, de données hydrométriques et de données piézométriques de 1972 à 1979 . L'objectif de cette étude menée avec le programme NEWSAM était de déterminer l'influence sur le débit de la rivière de pompages dans la nappe destinés au soutien du débit d'étiage, en vue d'assurer une alimentation suf- fisante d'une usine de traitement des eaux en aval. Il s'agissait d'un problème de relations nappe-rivière pour lequel étaient disponibles simultanément des données souterraines et des données de surface, c'est pourquoi il a paru intéressant de retenir ce bassin à titre de test du modèle couplé. Soulignons cependant que le ruissellement représente une faible partie de l'écoulement ; c'est d'ailleurs pourquoi il a d'abord été possible de traiter le problème avec le modèle souterrain NEWSAM.

L'étude au moyen du modèle intégré surface-souterrain étant actuellement en cours de réalisation nous ne sommes en mesure de présenter que le cadre de la modélisation.

\section{Schématisation multicouche}

Le modèle comprend :

- deux couches souterraines de 618 et 703 mailles représentant les nappes de la craie turonienne et cénomanienne,

- une couche de surface de 424 mailles recouvrant le bassin de la haute Lys sur $120 \mathrm{~km}^{2}$ et une partie des bassins de l'Aa, la Ternoise, la Planquette, la Laquette, la Créquoise et le Surgeon.

\section{Réseau de drainage}

Ayant choisi les mailles rivières sur le tracé de la Lys et de ses principaux affluents, on obtient 63 sous-bassins sur lesquels seront effectués les transferts de surface (Fig. 1).

Les étapes suivantes consisteront tout d'abord à tenter de reconstituer les débits naturels en cinq stations hydrométriques et les variations du niveau des nappes en trente stations piézométriques depuis l'année 1972. On dispose de plus, d'un essai de pompage avec soutien du débit en rivière qui sera également simulé. 


\section{Discussion}

Président : M.C. BOCQUILLON

M. Le Président. - M. LEDOUX nous a présenté un modèle qui doit résoudre de façon définitive tous les problèmes de l'hydrologie. Je pense que notre discussion sera certainement animée. $\mathrm{Y}$ a-t-il des intervenants?

M. THIRRIOT. - Vous avez parlé de 14 mécanismes de production. Pouvez-vous en dire quelques mots?

$M$. LEDOUX. - Le programme existant permet en effet de prendre en compte 14 mécanismes distincts pour la fonction production. Cependant, même en disposant de 14 mécanismes, il n'est pas possible à notre avis de traiter tous les cas rencontrés dans les applications pratiques. C'est pourquoi nous n'avons pas parlé ici du đétail des fonctions production employées, laissant à chaque utilisateur le choix le mieux adapté à son problème.

M. THIRRIOT. - Pourriez-vous donner quelques exemples?

M. LEDOUX. - Sur l'exemple du bassin de la Lys nous avons utilisé seulement 4 types de fonction production. Le plus simple est le mécanisme décrivant le comportement du sol imperméable que l'on peut rencontrer par exemple en zone urbaine. Dans ce cas l'infiltration est nulle et l'évapotranspiration réelle est prise dans la lame d'eau précipitée jusqu'à concurrence de l'évapotranspiration potentielle. Le mécanisme sol nu, également utilisé, fait appel à la notion classique de réserve facilement utilisable.

M. THIER Y. - Je voudrais savoir de quel type d'information piézométrique on a besoin pour caler un tel modèle.

M. LEDOUX. - D'une façon générale il faut utiliser les mêmes données que pour le calage d'un modèle classique de nappes, soit des données piézométriques et des données de débit. Les ajustements sont toutefois plus difficiles car il faut tenir compte simultanément de paramètres plus nombreux. Le modèle une fois calé inspirera cependant une plus grande confiance.

Sur le bassin de la Lys nous avons utilisé 4 stations de jaugeage fournissant les débits journaliers depuis 1972, ainsi que les mesures dans 45 piézomètres.

M. THIERY. - Plus précisément, je voudrais savoir quel est l'ordre de grandeur du pas de temps dont on doit disposer pour les mesures piézométriques. Le modèle fait en effet un bilan détaillé en régime transitoire faisant intervenir à la fois des phénomènes lents (infiltration) et rapides (ruissellement).

M. LEDOUX. - Les précipitations et les débits sont en général connus au pas de temps journalier suivant l'habitude en hydrologie. C'est moins courant pour les données piézométriques qui seront plutôt hebdomađaires ou même mensuelles. L'ajustement du modèle devra donc être fait compte-tenu de la fréquence des observations disponibles. Le programme peut toutefois s'adapter à n'importe quel pas de temps.
M. DUJARDIN. - La SOGREAH est en train d'étudier avec le Danish Hydraulic Institute et l'Institute of Hydrology de Wallingford un modèle Eaux de surface - Eaux souterraines.

Il faut, pour cela, harmoniser les méthodes des trois disciplines, hydrologie, hydraulique, hydrogéologie, et notamment imposer en surface un maillage peu compatible avec le réseau hydrographique.

Il se pose surtout le problème du réglage global d'un tel modèle. On s'en tire actuellement en réglant séparément les deux niveaux. On introduit les résultats du modèle de surface dans le modèle de nappe et on regarde si ce dernier peut être réglé avec ces données ; sinon on voit dans quelle marge d'erreurs on se situe et on règle progressivement les deux modèles par le contrôle de la cohérence des transferts d'un modèle à l'autre. Croyez-vous qu'on puisse régler globalement un tel modèle ?

Ce que vous faites est très intéressant, mais c'est un problème délicat que de règler plusieurs fonctions à la fois.

M. LEDOUX. - Notre approche est en fait tout à fait sem. blable. On essaye tout d'abord de règler indépendamment le maximum de paramètres de la surface et du souterrain avant de passer au couplage proprement dit qui n'intervient que dans le dernier programme. Il est cependant nécessaire à partir d'un certain moment de traiter le problème globalement ne serait-ce que pour résoudre les questions de non-linéarité.

M. CHÉRON. - Le modèle permet-il de tenir compte des phénomènes périodiques tels que les fluctuations de marée dans le cas de l'existence d'une relation entre le rivière et la nappe ? Outre ce cas, cela peut-il concerner les pompages d'eau qui peuvent être assimilés à des phénomènes quasi-cycliques.

M. LEDOUX. - Le modèle permet théoriquement de tenir compte des phénomènes périodiques. C'est en pratique un problème de discrétisation du temps et de l'espace. Sur l'exemple de la Lys les mailles situées au voisinage des rivières ont une taille d'environ $150 \mathrm{~m}$, ce qui n'est vraisemblablement pas à l'échelle des fluctuations semi-diurnes induites par la marée.

M. THIRRIOT. - Les fluctuations ne vont pas se faire sentir très loin. Il faudrait d'ailleurs faire intervenir des fluctuations autres que celles de la marée, par exemple celles dues à l’exploitation des puits.

Ces fluctuations peuvent être traitées comme un problème de couche limite avec une longueur de pénétration proportionnelle à $\sqrt{D T}, D$ étant la diffusivité équivalente, $T$ la pseudo période caractéristique des fluctuations.

$M$. Le Président. - Je vous remercie.

Nous allons passer à la communication suivante. 